\title{
Escala de Comportamentos de Bullying (ECB): Elaboração e Evidências Psicométricas
}

\author{
Emerson Diógenes de Medeiros - Universidade Federal do Piani, Parnaíba, Brasil \\ Valdiney Veloso Gouveia - Universidade Federal da Paraíba, João Pessoa, Brasil \\ Renan Pereira Monteiro - Universidade Federal da Paraíba, João Pessoa, Brasil \\ Paulo Gregório Nascimento da Silva - Universidade Federal do Vale do São Francisco, Petrolina, Brasil \\ Bruna de Jesus Lopes - Universidade Federal da Paraíba, João Pessoa, Brasil \\ Paloma Cavalcante Bezerra de Medeiros - Universidade Federal do Piani, Parnaiba, Brasil \\ Élido Santiago da Silva - Universidade Federal do Pianí, Parnaíba, Brasil
}

\begin{abstract}
Resumo
Este estudo objetivou elaborar a Escala de Comportamentos de Bullying (ECB), checando evidências de validade e precisão. Participaram 455 estudantes do ensino fundamental de escolas públicas e particulares de Parnaíba (PI), com idade média de 11,3 anos, em maioria do sexo feminino (53,5\%). Todos responderam à versão preliminar da medida, de 30 itens, e perguntas demográficas. Realizou-se a análise dos eixos principais (AEP) (rotação Varimax), o Scree Test, e a análise paralela indicou a existência de uma estrutura tetrafatorial. Logo, realizou-se uma nova AEP, fixando a extração de quatro fatores, estes explicaram conjuntamente $47,7 \%$ da variância total, cujos alfas de Cronbach variaram de 0,60 a 0,79. Esses achados revelam que a ECB reúne evidências favoráveis de validade e consistência interna, podendo ser adequadamente utilizada em estudos futuros para conhecer os antecedentes e consequências do bullying.
\end{abstract}

Palavras-chave: bullying, medida, validade

\section{Bullying Behaviors Scale (BBS): Development and Psychometrics Evidences}

\begin{abstract}
This work aimed to develop the Bullying Behaviors Scale (BBS), checking evidences of validity and reliability. 455 elementary school students from public and private schools in Parnaíba (PI), Brazil, participated in the study. Their mean age was 11.3, being mostly female $(53.5 \%)$. All participants completed a preliminary version of the measure, composed by 30 items, and demographic questions. A Principal Axis Analysis was performed (PAF; Varimax rotation), the Scree test, and parallel analysis indicated the existence of a four-factor structure, consistent with the literature (verbal, physical, relational and virtual). Next, a new PAF was performed, establishing the extraction of four factors, which account for $47.7 \%$ of total variance, and Cronbach alphas ranged from .60 to .79. These findings reveal that the BBS meets favorable evidence of validity and reliability, and it is suitable for use in future studies aiming to understand the antecedents and consequences of bullying.
\end{abstract}

Keywords: bullying, measure, validity

\section{Escala de Comportamientos de Bullying (BCE): Elaboración y Evidencias Psicométricas}

\section{Resumen}

Este estudio tuvo como objetivo elaborar la Escala de Comportamientos de Bullying (ECB), examinando evidencias de validez y precisión. Participaron 455 estudiantes de Enseñanza Primaria de escuelas públicas y privadas de la ciudad de Parnaíba (PI), Brasil, con edad media de 11,3 años, la mayoría del sexo femenino (53.5\%). Todos respondieron a la versión preliminar de la escala que contiene 30 ítems, y a preguntas demográficas. Se realizó el análisis de los Ejes Principales (AEP) (rotación Varimax), el Scree Test, y análisis paralelos, los cuales indicaron la existencia de una estructura de cuatro factores. Luego se realizó una nueva AEP fijando la extracción de cuatro factores, que explicaron el 47,7\% de la variabilidad total, y sus alfas de Cronbach variaron de 0,60 a 0,79 . Estos resultados ponen de manifiesto que la ECB reúne evidencias favorables de validez y precisión, y se puede utilizar adecuadamente en futuros estudios para conocer los antecedentes y las consecuencias del bullying.

Palabras clave: bullying, medida, validez

\section{Introdução}

Característico do ambiente escolar, o fenômeno bullying, apesar de ser prática antiga, passou a ganhar destaque no âmbito acadêmico apenas na década de 1970. Nesse cenário, cabe destacar os estudos pioneiros de Dan Olweus, considerando agressores e vítimas em contexto escolar (Bandeira, 2009; Sanders, 2004).

Ao longo do tempo, o bullying foi se estabelecendo como um construto independente, apresentando alguns aspectos peculiares que o definem, tais como a intencionalidade do agressor, a repetição dos atos, a 
assimetria de forças entre perpetrador e vítima, a vulnerabilidade desse último, mostrando incapacidade de se defender e a intenção do agressor em causar sofrimento no indivíduo vitimizado (Analitis et al., 2009; Olweus, 2011; Solberg, Olweus, \& Endresen, 2007).

Além de reforçar tais aspectos, Farrington (1993) cita diversas formas de expressão do bullying (e.g., física, verbal e psicológica). Não obstante, mais recentemente, com a constante evolução de tecnologias de comunicação, percebe-se o aumento de casos em ambiente virtual, constituindo-se como uma manifestação moderna desse construto, denominada de cyberbullying (Juvonen \& Gross, 2008; Slonje \& Smith, 2008).

Concretamente, considerando os aspectos encontrados na literatura, pode-se conceituar o bullying como uma forma de agressão física, verbal ou psicológica, difundida, principalmente, na escola, levada a cabo por crianças e adolescentes. Nessa direção, um aluno ou grupo pratica de forma repetida atos que podem causar prejuízo emocional, psicológico e social ao vitimizado que se encontra em uma relação desigual de poder, impedindo que se defenda das agressões sofridas (Baldry \& Farrington, 2000; Rolim, 2008; Salmivalli, 2010).

Considerando as manifestações do bullying, de acordo com Olweus (1993), destacam-se dois tipos: direto e indireto. O bullying direto é mais fácil de ser identificado, sendo composto por duas dimensões específicas: física (e.g., bater, roubar, danificar objetos da vítima) e verbal (e.g., agressões verbais, apelidos desagradáveis, ameaças e xingamentos). É importante ressaltar que estudos (Bender \& Lösel, 2011; Wal, Wit, \& Hirasing, 2003) apontam a relação de tais comportamentos com predisposição à agressividade e à violência, além de outras condutas antissociais, como uso de drogas. A manifestação indireta, por sua vez, é mais difícil de detectar. Por ser menos explícita, é pouco visível aos educadores e responsáveis (Ribeiro, 2007). Expressões do bullying indireto envolvem, principalmente, aspectos de ordem relacional, como, por exemplo, ações de exclusão grupal e disseminação de boatos (Monks \& Coyne, 2011).

Apesar de diferirem quanto a suas formas de expressão, ambas são extremamente prejudiciais às vítimas, sobretudo quando juntas. Nesse sentido, é possível destacar que vitimizados(as) por formas diretas e indiretas do bullying mostram-se mais vulneráveis a desenvolverem problemas comportamentais futuros, a exemplo do uso de drogas ou envolvimento em comportamentos antissociais e delitivos, quando comparados à exposição isolada a um desses tipos (Wolke, Woods, Blomfield, \& Karstadt, 2000).

Mais recentemente, tem se destacado uma nova forma de expressão do bullying, reflexo do avanço das tecnologias da informação, como apontado previamente, nomeado como cyberbullying (Jimerson, Swearer, \& Espelage, 2010). Essa nova expressão está em evidência, muito em função de acidentes pessoais, a exemplo de suicídios relacionados à divulgação de vídeos ou imagens por meio da internet ou smartphones (Juvonen \& Gross, 2008; Slonje \& Smith, 2008).

Essa recente tipologia pode ser considerada uma evolução na manifestação do próprio bullying (Maidel, 2009), sendo expresso de quatro maneiras: 1) agressão por meio de mensagem de texto; 2) e-mails; 3) ligações telefônicas e 4) postagens, na internet ou via celulares, de vídeos e fotos que possam causar danos à vítima (Slonje \& Smith, 2008; Smith et al., 2008). Logo, o agressor tem uma variedade de ferramentas à disposição para ofender e denegrir a imagem de seus pares (Juvonen \& Gross, 2008).

Quanto a sua caracterização, o cyberbullying diferencia-se dos demais em um aspecto, nele não há disparidade de forças (Maidel, 2009; Vandebosch \& Van Cleemput, 2008), ou seja, o agressor não necessariamente precisa ser superior físico e/ou mentalmente à vítima, pois os cyberbullies escondem-se no anonimato (Smith et al., 2008).Contudo, existem pesquisas que desconsideram tal suposição, compreendendo a vida on-line como uma extensão da vida escolar, mantendo, assim, os mesmos padrões de relação (Juvonen \& Gross, 2008). Nessa direção, em estudo realizado por Kwan e Skoric (2013), relacionando o cyberbullying à forma tradicional, foram encontrados resultados que indicam correlações positivas entre ser agressor $(r=0,56, p<0,001)$ e vítima $(r=0,28, p<0,001)$ em ambos os tipos (cyber e tradicional)". Cabe destacar que um agravo do cyberbullying, comparado ao tradicional, refere-se à ampliação ilimitada das testemunhas às agressões (Juvonen \& Gross, 2008).

É possível, portanto, identificar algumas formas de expressão do bullying que, ocorrendo em conjunto ou separadamente, costumam ter graves consequências [e.g., dificuldades acadêmicas, depressão e risco de ideação suicida (Arseneaultet al., 2006; Holmberg \& Hjern, 2008)]. Não obstante, verificam-se efeitos distintos que o bullying causa nos diferentes grupos envolvidos, nessa direção, parece algo pertinente descrever os atores sociais envolvidos em tais condutas, indicando potenciais correlatos de cada um. 


\section{Tipo de Envolvimento e seus Correlatos}

Segundo Olweus (2003), existem quatro tipos de grupos envolvidos em atos de bullying: agressores, vítimas, agressores/vítimas e testemunhas. Os agressores são aqueles que tentam assumir a liderança em grupos, valendo-se de agressões, buscando reafirmar-se sobre os mais frágeis, físico e psicologicamente (Ferraz, 2008). Estudos indicam que os bullies podem apresentar, ao avançar da idade, distúrbios psiquiátricos, a exemplo de déficit de atenção, depressão e conduta de desordem (Kokkinos \& Panayiotou, 2004; Kumpulainen, Räsänen, \& Puura, 2001), ou ainda vir a apresentar problemas escolares, consumo de drogas e comportamentos violentos (Baldry \& Farrington, 2000; Bender \& Lösel, 2011; Farrington \& Ttofi, 2009; Méndez \& Cerezo, 2010).

As vítimas, por sua vez, podem ter prejuízos desenvolvimentais em distintos domínios (e.g., social, acadêmico e emocional; Pinheiro, 2006). No caso, estudos apontam associação entre o sujeito vitimizado e problemas, como depressão, tendência à ideação suicida, timidez e introversão, decréscimo em habilidades sociais (Fleming \& Jacobsen, 2009; Klomek, Sourander, \& Gould, 2010; Moura, Cruz, \& Quevedo, 2011; Owusu, Hart, Oliver, \& Kang, 2011; Skapinakiset al., 2011).

Por sua vez, os agressores/vítimas envolvem-se nos dois cenários, revidam agressões, constituindo uma tentativa de prevenir futuras ações dos bullies, ou encontram indivíduos mais vulneráveis para quem são agressores, numa tentativa de fortalecer sua própria imagem (Fante, 2005; Ireland \& Archer, 2004; Wal, 2005). Esse grupo, de acordo com a literatura consultada, mostra-se mais propenso a exibir condutas delinquentes e agressivas, baixos índices de comportamento pró-social (Arseneault et al., 2006; Holmberg \& Hjern, 2008; Kim, Koh, \& Leventhal, 2005; Sourander et al., 2007).

Por fim, as testemunhas, que representam a maioria, participam das situações de bullying apenas como espectadores, não se manifestando por medo de sofrerem represálias e se tornarem alvos (Fosse, 2006; Lopes Neto, 2005). Esse grupo apresenta um papel importante, pois, com a observação e o consentimento destes, o bullying persiste e o ato se torna um processo realizado em grupo (Almeida, 2009; Fonseca \& Veiga, 2007).

Resumidamente, estima-se que o bullying pode ser uma variável antecedente de diversos problemas, com efeitos imediatos ou tardios, tanto para quem é vítima quanto para quem agride (Sourander et al., 2007). Desse modo, assevera-se que o bullying tornou-se um problema de saúde pública (Kim et al., 2005), podendo acarretar diversos problemas biopsicossociais aos envolvidos (Lemos, 2007).

Diante do exposto, evidencia-se a necessidade de identificar, por meio de instrumentos avaliativos eficazes, pessoas que estão envolvidas em situações de bullying, seja no papel de agressor ou vítima. Estimando precisamente a prevalência desse fenômeno, é possível subsidiar estratégias de combate e prevenção dessa forma de agressão entre pares, buscando desenvolver, assim, um ambiente sadio e pacífico que possibilite melhor convivência, especialmente entre os escolares. Diante dessa conjuntura, o presente estudo objetivou elaborar uma medida que permita identificar agressores em atos de bullying. Não obstante, antes de seguir para o estudo empírico, cabe citar algumas medidas que vêm sendo propostas para avaliação do bullying, algo tratado no tópico a seguir.

\section{Avaliação do bullying}

Em revisão sistemática, buscando avaliar a qualidade metodológica de estudos que trataram das propriedades psicométricas de medidas de bullying, Vessey, Strout, DiFazio e Walker (2014) encontraram 31 estudos, que se utilizaram de 25 medidas de bullying, avaliando diferentes grupos e fatores específicos. Por exemplo, os autores citam medidas que avaliam especificamente a vitimação do bullying (e.g., California Bullying Victimization Scale; Felix, Sharkey, Green, Furlong, \&Tanigawa, 2011), que avaliam simultaneamente agressores e vítimas (e.g., Formsof Bullying Scale; Shaw, Dooley, Cross, \& Zubrick, 2013) e instrumentos que focam no cyberbullying (e.g., E-Victimization e E-Bullying Scale; Lam \& Li, 2013).

Em contexto brasileiro, a realidade é algo distinta quanto à construção e adaptação de instrumentos relacionados ao bullying, algo verificado no estudo de Alckmin-Carvalho, Izbicki, Fernandes e Melo (2014), que objetivaram realizar um levantamento dos instrumentos utilizados para estimar o bullying. No caso, os autores consideraram 25 artigos, verificando que, em 13 deles, foram utilizados instrumentos padronizados em outros países, contudo, sem evidências psicométricas no Brasil. Outros cinco estudos mediram o bullying por meio de situações, entretanto, estas não foram especificadas. Em dois artigos, os autores detectaram o bullying por meio de observação direta ou participante. Em outros três, utilizou-se, além de observação, a aplicação de questionário e, por fim, Alckmin-Carvalho et al. encontraram um estudo que avalia o bullying por meio 
de uma técnica projetiva e outro em que é apresentada uma definição de bullying, seguido de uma pergunta sobre a participação como agressor ou vítima. Dessa forma, evidenciou-se necessidade de instrumentos e estudos que apontem seus parâmetros psicométricos no Brasil.

Contudo, verificam-se esforços iniciais, a exemplo da adaptação da California Bullying Victimization Scale, levada a cabo por Soares (2013), checando evidências de validade fatorial e consistência interna. Ademais, Soares (2013) elaborou e testou uma medida que avalia as atitudes frente a potenciais alvos de bullying. Em outro estudo, Santos, Gouveia, Soares, Cavalcanti e Gouveia (no prelo) verificaram a validade de construto da Forms of Bullying Scale, encontrando índices que atestam a adequação da medida (e.g., GFI $=0,90$ e RMSEA $=0,06$ ).

Diante do exposto, verifica-se que mensurar o bullying vem sendo foco de intenso debate, muito em virtude das suas distintas formas de expressão e por envolver diferentes atores sociais (Shaw, Dooley, Cross, Zubrick, \& Waters, 2013; Thomas, Connor, \& Scott, 2015). Como reflexo desse cenário, observa-se a existência de medidas que avaliam conjuntamente agressores e vítimas, que focam, nas vítimas, outras avaliam isoladamente cyberbullying ou o bullying direto em detrimento do indireto, não contemplando as múltiplas formas de expressão do fenômeno. Além disso, é importante considerar as formas de expressão do bullying em fatores específicos, de modo que cada um pode apresentar correlatos distintos, bem como diferirem entre os sexos (Carbone-Lopez, Esbense, \& Brick, 2010; Rivers \& Smith, 1994).

Ressalta-se que avaliar o bullying como um todo envolveria uma abordagem multimétodo, utilizando medidas de autorrelato para identificar agressores e vítimas, bem como colhendo o relato dos pares e professores, além de realizar observações em situação de sala de aula e/ou nos intervalos. Contudo, a proposta aqui apresentada é de construir e conhecer evidências preliminares de validade fatorial e consistência interna de uma medida para identificar bullies, considerando a carência de estudos dessa natureza em contexto brasileiro (Alckimin-Carvalho, Izbicki, Fernandes, \& Melo, 2014). Ademais, diferente de outras propostas, buscou-se contemplar as formas direta (física e verbal) e indireta (relacional) de maneira equânime, bem como o cyberbullying, considerando a relevância de avaliar essa dimensão moderna junto com a forma tradicional (Thomas et al., 2015).

\section{Método}

\section{Participantes}

Para lograr os objetivos propostos, contou-se com uma amostra não probabilística (acidental) de 455 estudantes com idades entre 8 e 17 anos $(m=11,36$; $d p=$ 1,52), havendo prevalência de crianças com $11(29,9 \%)$, $10(25,7 \%)$ e $12(18 \%)$ anos. Os demais participantes dividiram-se da seguinte forma: 8 (1,1\%), 9 (4,9\%), 13 (8,9\%), 14 (7,5\%), $15(3,8 \%)$ e $17(0,2 \%)$ anos. Parece pertinente e justificado tal amplitude de idade, já que existem evidências de que o bullying é recorrente em crianças muito jovens, isto é, com idades iguais ou superiores a oito anos (Schoffstall \& Cohen, 2011). Em sua maioria, os participantes eram do sexo feminino $(53,5 \%)$ e provenientes de escolas públicas $(65,8 \%)$ da cidade de Parnaíba-PI. Ao serem indagados, 15,4\% indicaram cometer bullying e 23,8\% relataram serem vítimas de bullies, em algum momento. Por fim, 55,9\% dos participantes apontaram ter acesso à internet e 37,6\% indicaram que possuem perfis ativos em redes sociais (Facebook etc.).

\section{Instrumentos}

Os respondentes receberam um livreto contendo a versão preliminar da Escala de Comportamentos de Bullying (ECB), composta por 30 itens respondidos numa escala tipo Likert de cinco pontos, que representam a frequência de emissão de determinados comportamentosde bullying na última semana $(0=$ Nenhuma vez a $4=$ Quatro ou mais vezes por semana). Os participantes ainda tinham em conta a frase estímulo "em minha escola, ultimamente, apresentei tais comportamentos em relação aos meus colegas". Além da $\mathrm{ECB}$, responderam perguntas de caráter demográfico para caracterização da amostra [e.g., sexo, idade, tipo de escola (pública ou particular)].

\section{Procedimento}

Inicialmente, com fim de elaborar os itens, foram aplicados questionários abertos a estudantes de licenciaturas (Matemática, Pedagogia e Biologia) e Psicologia de uma Instituição de Ensino Superior pública, de Parnaíba-PI. Após aceitarem colaborar com o estudo, por meio da assinatura do Termo de Consentimento Livre e Esclarecido (TCLE), os estudantes registraram comportamentos associados às dimensões teorizadas. Originaram-se, então, 120 comportamentos (itens) considerados de bullying, que logo foram submetidos a uma análise de juízes. No caso, entrou-se em contato 
com sete profissionais da Psicologia e Pedagogia que, após consentirem sua participação, foram solicitados a classificar os itens/comportamentos em função das quatro dimensões teorizadas de bullying, que eram previamente esclarecidas em formulário próprio. Os itens que apresentaram consenso de 100\% entre os juízes foram retidos para formar a versão preliminar da ECB de trinta itens.

Com a versão preliminar, realizou-se a validação semântica, buscando eliminar itens que podiam ser confusos; partindo da leitura atenta de um grupo de 10 alunos, cinco de uma escola pública e outros cinco de escola particular, com idades de oito e nove anos. Nessa direção, contataram-se pais e responsáveis, onde foi enviado o TCLE solicitando a autorização para que as crianças participassem dessa etapa do estudo. No que tange à validação semântica, nenhuma alteração foi proposta pelos estudantes nesse estágio de desenvolvimento da escala.

Finalmente, após a aprovação do comitê de ética (CAAE: 0193.0.045.000-11), realizou-se contato com as direções das escolas, tanto públicas quanto particulares, para solicitação da autorização para a realização das coletas dos dados. Por se tratar de uma amostra composta por menores, após a autorização dos diretores das escolas, foi enviado aos pais e responsáveis um TCLE para que estes autorizassem suas crianças a participarem do estudo. Enfatizou-se, no próprio TCLE e instrumento de coleta, que a participação não traria nenhum prejuízo ou bônus ao respondente e que sua participação seria voluntária, podendo desistir de responder a qualquer momento sem prejuízos. Ainda buscou-se indicar que, em nenhum momento, os participantes seriam identificados no projeto, que as análises seriam tomadas no conjunto e somente teriam acesso aos dados os pesquisadores diretamente envolvidos com o projeto.

A coleta de dados foi realizada em ambiente coletivo (sala de aula), no entanto, os instrumentos foram respondidos de forma individual, em que sempre esteve presente um ou dois aplicadores para dirimir eventuais dúvidas dos partícipes. Aproximadamente, 20 minutos foram suficientes para responderem ao livreto de instrumentos.

\section{Análise de Dados}

Para a análise de dados, utilizou-se do pacote estatístico PASW, em sua versão 18. Mais especificamente, realizaram-se estatísticas descritivas (medidas de tendência central e dispersão) para caracterização da amostra, teste t de Student para se analisar o poder discriminativo dos itens, análise fatorial exploratória, alfa de Cronbach e homogeneidade, para verificar, respectivamente, a validade e fidedignidade da medida e, por fim, MANOVA, objetivando verificar se existem diferenças nos comportamentos de bullying em função do sexo dos participantes.

\section{Resultados}

Os resultados serão apresentados em subtópicos, em função da análise estatística empregada e o objetivo para qual se propõe. Desse modo, inicia-se com a subseção que trata da discriminação dos itens.

\section{Poder Discriminativo dos Itens}

A fim de verificar se os itens discriminam sujeitos com pontuações próximas, procedeu-se tal análise. Teve-se, em conta, a pontuação total dos participantes, a partir do critério interno da mediana, formaram-se os grupos critérios (inferior e superior). Posteriormente, as médias dos grupos foram comparadas para cada item do instrumento por meio de um teste $\mathrm{t}$ - Student para amostras independentes.

Todos os itens discriminaram satisfatoriamente os indivíduos com pontuações altas e baixas na $\mathrm{ECB}(t>$ 1,96; $p<0,05)$. Desse modo, com a qualidade métrica dos itens individualmente assegurada, partiu-se para a próxima etapa, conhecer evidências de validade e precisão do instrumento.

\section{Validade e Precisão}

Para se verificar a estrutura fatorial, optou-se por realizar uma análise fatorial exploratória (AFE). Para tanto, inicialmente, foi verificado a adequação dos dados ao tratamento multivariado. Os resultados iniciais apoiaram a realização de uma AFE $(K M O=0,87$ e o Teste de Esfericidade de Bartlett, $\chi^{2}=4002,941$; $p<0,001)$.

Nesse sentido, optou-se pelo método de extração dos eixos principais (principal axis factoring; PAF), sendo utilizada rotação varimax. Essa escolha se pautou em dois aspectos: (a) facilidade de interpretação da estrutura (Damásio, 2012) e (b) possibilidade de considerar independentemente os quatro tipos de bullying (Farrington, 1993; Juvonen \& Gross, 2008; Slonje \& Smith, 2008). Nesse caso, apesar de existirem evidências de que os fatores desse construto podem ter correlação moderada entre eles, não necessariamente a pessoa que apresenta comportamentos de um tipo bullying o fará 
em relação aos demais. O critério de Kaiser indicou a existência de sete possíveis fatores com autovalores superiores a um, no entanto, optou-se por observar o critério de Cattel (ver Figura 1) que indicou uma solução com quatro fatores.

Observa-se, na figura, que, ao traçar uma linha (pontilhada) passando pelo maior número de autovalores (círculos), quatro fatores se destacam em relação aos demais. Entretanto, para dirimir qualquer dúvida referente à quantidade de dimensões a serem extraídas, optou-se por realizar uma análise paralela (critério de Horn; Hayton, Allen, \& Scarpello, 2004), por ser mais robusta que os critérios de Kaiser e de Cattell, utilizados previamente.

Tal análise compara os autovalores empíricos com autovalores médios de 1.000 bancos de dados gerados aleatóriamente, com as mesmas características do banco empírico, ou seja, tamanho da amostra e número de itens do instrumento. Ao comparar os autovalores, se o observado for maior que o simulado, há o apoio à existência do fator.

O resultado dessa análise apoia a extração de quatro fatores, já que os primeiros quatro autovalores da AFE $(8,26 ; 2,96 ; 1,64$ e 1,43) foram superiores aos seus correspondentes gerados na análise paralela $(1,51 ; 1,43$; 1,38 e 1,34), o contrário acontece após o quinto autovalor $(1,28<1,30)$. De posse do apoio da literatura e critérios empíricos, rodou-se uma nova PAF, restringindo a extração de quatro fatores.

As dimensões explicaram conjuntamente 47,6\% da variância total. Foi considerado como carga fatorial mínima $|0,30|$ para que o item fosse retido no fator (Hair, Black, Babin, Anderson, \& Tatham, 2009; Pasquali, 2003, 2012). Ainda foram desconsiderados os itens que, mesmo com cargas fatoriais superiores ao mínimo aceitável, tinham conteúdo semântico diferente dos itens com maiores cargas fatoriais dentro do mesmo fator. Com o fim de apresentar um instrumento curto, que preze a parcimônia, optou-se, ainda, por escolher os quatro melhores itens de cada dimensão, para compor a versão final da ECB. Os resultados podem ser vistos na Tabela 1 a seguir.

O primeiro fator, denominado Bullying verbal, inicialmente, se considerando a carga fatorial mínima, englobaria onze itens, no entanto, os itens 5 (Colocar obstáculos para colegas tropeçarem) e 2 [Dar puxões (cabelo, roupa, etc.)] foram eliminados por obterem carga superior a $|0,30| \mathrm{em}$ mais de um fator, enquanto que os itens 9 (Dar tapas em colegas), 4 (Jogar objetos em colegas), 24 (Esbarrei propositalmente em colegas), 1 (Empurrei colegas) e 10 (Dei socos ou esmurrei um colega), por serem com semântica distinta ao conteúdo do fator, determinado pelos itens de maior carga fatorial, foram eliminados. Nesse sentido, o primeiro fator

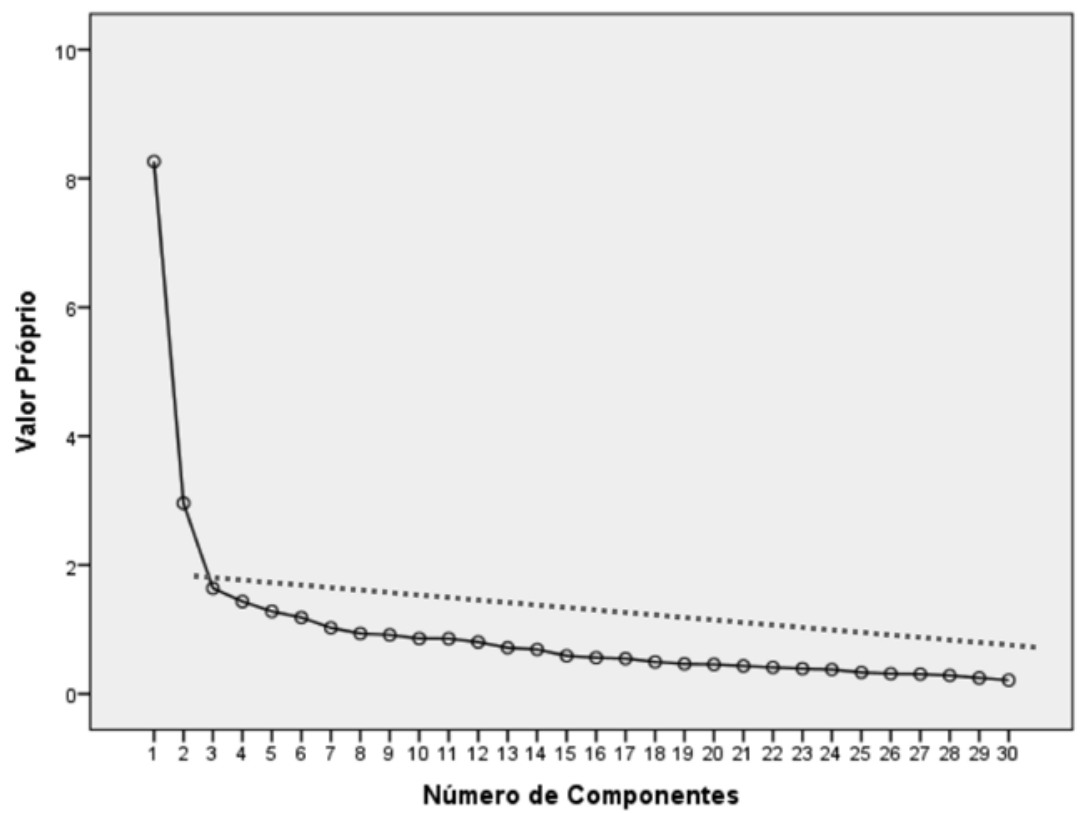

Figura 1. Representação gráfica dos valores próprios. 
Tabela 1

Estrutura Fatorial da Escala de Comportamentos de Bullying (ECB)

\begin{tabular}{|c|c|c|c|c|c|}
\hline \multirow[b]{2}{*}{ Item resumido } & \multicolumn{4}{|c|}{ Fatores } & \multirow[b]{2}{*}{$h^{2}$} \\
\hline & BV & $\mathrm{CB}$ & $\mathrm{BF}$ & BR & \\
\hline 15. Apelidar colegas & $0,73^{*}$ & 0,12 & 0,08 & 0,27 & 0,64 \\
\hline 12. Falar com tom de voz agressivo com um colega & $0,72 *$ & 0,22 & 0,10 & 0,06 & 0,58 \\
\hline 14. Fazer piadas de mau gosto & $0,72 *$ & 0,09 & 0,06 & 0,20 & 0,57 \\
\hline 11. Falar mal de colegas & $0,70^{*}$ & 0,13 & 0,14 & $-0,01$ & 0,53 \\
\hline 9. Dar tapas em colegas & 0,69 & 0,09 & 0,13 & 0,28 & 0,58 \\
\hline 1. Empurrei colegas & 0,60 & 0,05 & 0,20 & 0,06 & 0,40 \\
\hline 4.Jogar objetos em colegas & 0,57 & $-0,05$ & 0,18 & 0,13 & 0,39 \\
\hline 5. Colocar objetos para que tropessasem & 0,52 & 0,23 & 0,38 & 0,15 & 0,50 \\
\hline 24. Esbarrei propositalmente em colegas & 0,52 & 0,20 & 0,04 & 0,28 & 0,39 \\
\hline 2. Dar puxões (cabelo, roupa, etc.) & 0,47 & 0,05 & 0,43 & 0,11 & 0,43 \\
\hline 10. Dar socos ou esmurrar colegas & 0,44 & 0,16 & 0,34 & 0,30 & 0,44 \\
\hline 23. Publicar fotos, na internet, ridicularizando colegas & 0,02 & $0,82^{*}$ & 0,03 & 0,10 & 0,68 \\
\hline 30. Publicar montagens na internet ridicularizando colegas & 0,05 & $0,71 *$ & 0,11 & 0,28 & 0,60 \\
\hline 28. Postar, na internet, vídeos constrangedores de colegas & $-0,05$ & $0,71^{*}$ & $-0,01$ & 0,13 & 0,51 \\
\hline 27. Criar grupos ou comunidades para ridicularizar colegas & 0,09 & $0,71 *$ & 0,28 & $-0,11$ & 0,59 \\
\hline 26. Hackiar perfis em redes sociais & 0,13 & $0,65^{*}$ & 0,05 & $-0,01$ & 0,44 \\
\hline 25. Insultar colegas via SMS e/ou e-mail & 0,23 & $0,63^{*}$ & $-0,02$ & 0,13 & 0,47 \\
\hline 29. Excluir colegas de comunidades virtuais & 0,20 & $0,56^{*}$ & 0,19 & 0,01 & 0,39 \\
\hline 21. Fazer comentários ofensivos em fotos na internet & 0,34 & $0,58^{*}$ & $-0,20$ & 0,07 & 0,27 \\
\hline 8. Abaixar as calças de um colega em público & 0,23 & 0,10 & $0,68^{*}$ & 0,00 & 0,52 \\
\hline 22. Criar fakes de colegas em redes sociais & 0,07 & 0,20 & 0,61 & $-0,07$ & 0,42 \\
\hline 6. Rasgar roupas e/ou quebrar objetos & 0,03 & 0,03 & $0,57 *$ & 0,21 & 0,37 \\
\hline 16. Expor publicamente colegas a situações constrangedoras. & 0,30 & $-0,06$ & 0,50 & 0,22 & 0,40 \\
\hline 7. Pisar em colegas propositalmente & 0,19 & 0,37 & $0,44^{*}$ & 0,35 & 0,49 \\
\hline 3. Chutar ou dar pontapés em colegas & 0,42 & 0,00 & $0,43^{*}$ & 0,32 & 0,47 \\
\hline 19. Excluir ou convencer amigos a excluírem colegas & 0,13 & 0,07 & 0,28 & $0,66^{*}$ & 0,53 \\
\hline 17. Insultar colegas em por andarem com colegas do sexo oposto & 0,17 & 0,04 & 0,06 & $0,62^{*}$ & 0,43 \\
\hline 18. Isolar colegas por apresentarem certas características & 0,20 & 0,03 & $-0,13$ & $0,60^{*}$ & 0,42 \\
\hline 20. Insultar colegas em função de seus amigos & 0,12 & 0,21 & 0,25 & $0,59 *$ & 0,47 \\
\hline 13. Fazer colegas chorarem por algo que disse & 0,30 & 0,00 & 0,29 & 0,36 & 0,31 \\
\hline Número de itens & 4 & 4 & 4 & 4 & \\
\hline Variância explicada & $27,5 \%$ & $9,8 \%$ & $5,7 \%$ & $4,7 \%$ & \\
\hline Alfa de Cronbach & 0,79 & 0,73 & 0,62 & 0,60 & \\
\hline
\end{tabular}

Nota. $*$ Item retido no fator; $\mathrm{BV}=$ bullying verbal; $\mathrm{CB}=$ cyberbullying; $\mathrm{BF}=$ bullying físico; $\mathrm{BR}=$ bullying relacional. 
ficou composto por quatro itens e apresentou autovalor igual a 8,26 , explicando $27,5 \%$ da variância total, as cargas fatoriais variaram de 0,70 (item 11 - Falar mal de colegas) a 0,73 (item 15 - Apelidar colegas), a precisão apresentou-se adequada [alfa de Cronbach de 0,79 e índice de homogeneidade (correlação média inter-itens $/ \mathrm{r}_{\mathrm{m} . \mathrm{i}}$ ) de 0,48$]$.

O segundo fator, nomeado de Cyberbullying, inicialmente contava com oito itens, optou-se pela permanência dos quatro com maiores saturações, objetivando tornar o instrumento mais parcimonioso. Observa-se que esse componente teve um autovalor de 2,96, explicando $9,8 \%$ da variância total; seus quatro itens possuiram cargas fatoriais variando de 0,70 (item 27 - Criar grupos ou comunidades para ridicularizar colegas) a 0,82 (item 23 - Publicar fotos na internet ridicularizando colegas), apresentando alfa de Cronbach igual a 0,73 e $r_{\mathrm{m} . \mathrm{i}}=0,44$.

O terceiro fator, denominado Bullying físico, possui autovalor igual a 1,64, explicando $5,4 \%$ da variância total, com saturações variando de 0,43 (item 3 - Chutar ou dar pontapés em colega) a 0,68 (item 8 - Abaixar as calças de um colega em público). Esse seria composto, inicialmente, por seis itens, não obstante, foram excluídos os itens 22 (Criar fakes de colegas em redes sociais) e 16 (Expor publicamente colegas a situações constrangedoras) por serem semanticamente distintos ao fator, e o último possuir escore fatorial aceitável em mais de uma dimensão. Logo, quatro itens formaram esse componente, possuindo fidedignidade aceitável (alfa de Cronbach igual a 0,62 e $r_{\mathrm{m}, \mathrm{i}}=0,30$ ).

O quarto fator apresentou autovalor de 1,43, explicando $4,8 \%$ da variância total, sendo denominado de Bullying relacional. Esse fator foi composto, inicialmente, por quatro itens com cargas fatorais que variavam de 0,59 (item 20 - Insultar colegas em função de seus amigos) a 0,66 (item 19 - Excluir ou convencer amigos a excluírem colegas), sua precisão está dentro do aceitável para uso em pesquisas (alfa de Cronbach foi de 0,60 e $r_{\mathrm{m} . \mathrm{i}}=0,31$ ). Considerando o bullying como um construto unifatorial, checou-se a precisão de todos os itens saturando em um fator geral, sendo possível observar índices aceitáveis que atestam a consistência interna da medida $\left(r_{\mathrm{m} . \mathrm{i}}=0,25 \mathrm{e} \alpha=0,83\right)$.

Por fim, verificou-se que os comportamentos de bullying se diferenciam em função do sexo dos participantes, havendo maiores médias para o grupo de meninos em todas as formas de agressão (lambda de Wilkis $=0,95 ; F(4,362)=4,604 ; p<0,001)$. Especificamente, tendo em conta o tamanho do efeito, percebe-se que as maiores diferenças entre meninos e meninas se deram no bullying físico $\left(\mathrm{n}^{2} \mathrm{p}=0,04\right)$, ao passo que a única dimensão que não diferenciou os participantes em função do sexo foi o bullying relacional $\left(\eta^{2} \mathrm{p}=0,01\right)$.

\section{Discussão}

$\mathrm{Na}$ literatura nacional, não foram encontrados instrumentos que avaliem os comportamentos de agressores em atos de bullying pautados nas quatro formas de expressão do construto. Recentemente, Soares (2013) validou a California Bullying Victimization Scale, não obstante, tal medida tem como foco a vítima, e não o agressor. Santos et al. (no prelo) também verificaram os parâmetros psicométricos de uma medida de bullying, a Formsof Bullying Scale, contudo, esta avalia agressor e vítima por meio de um mesmo instrumento, além de não cobrir as diversas formas de expressão desse construto em fatores específicos, algo necessário de se ter em conta, pois são consistentes os estudos que indicam um padrão distinto entre meninos e meninas com relação ao bullying físico, verbal e relacional, tal como evidenciado nessa ocasião (Carbone-Lopez et al., 2010; Rivers \& Smith, 1994). Nessa direção, planejou-se levar a cabo o presente estudo, objetivando elaborar e conhecer evidências preliminares de validade fatorial e consistência interna da Escala de Comportamentos de Bullying (ECB).

Avalia-se que os objetivos propostos foram alcançados, pois os resultados encontrados nesta pesquisa apontam propriedades psicométricas aceitáveis da ECB, além de contar com o apoio teórico, indicando a pertinência de se considerar o bullying como um construto formado por quatro dimensões distintas (Scheithauer, Hayer, Petermann, \& Jugert, 2006; Wang, Jannotti, \& Nansel, 2009; Wolke et al., 2000).

Especificamente, seus parâmetros psicométricos estão dentro dos recomendados pela literatura (Pasquali, 2003, 2010, 2012; Urbina, 2007). No tocante, a confiabilidade dos fatores, além do alfa de Cronbach, sensível ao número de itens, teve-se em conta a correlação média interitens. Verificou-se que os resultados para esse parâmetro estão dentro dos aceitáveis para fins de pesquisa, apesar de dois fatores apresentarem alfas próximos ao límite aceitável $(\alpha=0,60)$, algo que pode ser reflexo dos poucos itens que os formam. Contudo, ao se avaliar a homogeneidade, verificaramse valores adequados $\left(r_{\mathrm{m} . \mathrm{i}}>0,30\right.$; Clark \& Watson, 1995; Nunnally, 1991). Ademais, a pontuação total da ECB apresentou precisão igual, ou superior, ao de 
outras medidas de avaliação do bullying (Fitzpatrick \& Bussey, 2011; Shaw et al., 2013; Santos et al., no prelo; Soares, 2013).

A análise fatorial (PAF), com o apoio da Análise Paralela, permitiu averiguar que a ECB é uma medida claramente tetrafatorial, avaliando comportamentos de bullying em suas manifestações direta (física e verbal), indireta (relacional) e virtual (cyberbullying). Especificamente, verificou-se que as saturações foram acima do ponto de corte preconizado pela literatura $|0,30|$ (Hair et al., 2009; Pasquali, 2012), indicando que o instrumento apresenta evidências de validade fatorial.

Entretanto, este estudo apresenta algumas limitações, a exemplo do viés da amostragem não probabilística, não refletindo a composição real da população, e tamanho amostral, que não possibilitou averiguar a pertinência dos itens para todas as idades dos participantes, em especial os mais jovens, o que fugia ao escopo deste estudo. Entretanto, destacase que há indícios de que crianças, mesmos as muito jovens, como as aqui utilizadas, já são protagonistas e/ ou vítimas de cyberbullying (Schoffstall \& Cohen, 2011). Outro ponto importante a se destacar é a desejabilidade social, que leva os sujeitos a responderem de modo a revelar seus traços mais favoráveis ou socialmente aceitos. Esse problema é comum em pesquisas que tratam de assuntos delicados, a exemplo de comportamento agressivo e violento (Cozby, 2003).

A despeito de possibilidades futuras, é importante controlar o falseamento das respostas, utilizando, por exemplo, uma medida de desejabilidade social que controle esse viés. Ademais, é importante que esta pesquisa seja replicada em outros contextos. Amostras maiores e mais heterogêneas são essenciais para aumentar as evidências em torno da ECB, incluindo-se pessoas de diferentes níveis de escolaridade, classes sociais ou ambientes de moradia (urbano/ rural; capital/interior). Sugere-se, igualmente, empregar procedimentos de modelagem por equações estruturais, com o propósito de confirmar a estrutura fatorial da ECB ou mesmo testar modelos alternativos, como um trifatorial, agrupando os itens relativos ao bullying físico e verbal em um único fator, representando a forma direta de expressão. No mesmo sentido, parece útil checar a invariância fatorial dessa medida em diferentes grupos (e.g., homens e mulheres, escolas públicas e privadas, interior e capital).

No que tange à precisão da ECB, futuramente pode-se estimá-la por meio do teste-reteste, além de verificar a validade convergente e preditiva. Neste último caso, o bullying poderia ser avaliado por autorrelato e, posteriormente, informado por pares e professores, buscando verificar se, de fato, a ECB consegue estimar precisamente os agressores em atos de bullying.

Pode-se pensar, ainda, em pesquisas que objetivem conhecer potenciais antecedentes e consequentes dos atos de bullying, visto que esse padrão comportamental relaciona-se empiricamente com diversos construtos, tais como depressão e comportamentos antissociais e delitivos (Bender \& Lösel, 2011; Farrington \& Ttofi, 2009).

Em resumo, reuniram-se evidências suficientes da adequação psicométrica da Escala de Comportamentos de Bullying. Compreende, portanto, um instrumento breve, composto por 16 itens (quatro para cada dimensão), de fácil compreensão, com itens curtos, que pode ser usado adequadamente em estudos em que o interesse é conhecer os antecedentes e consequentes de condutas de bullying.

\section{Referências}

Alckmin-Carvalho, F., Izbicki, S., Fernandes, L. F. B., \& Melo, M. H. S. (2014). Estratégias e instrumentos para a identificação de bullying em estudos nacionais. Avaliação Psicológica, 13, 343-350. Recuperado de http://pepsic.bvsalud.org/pdf/avp/v13n3/ v13n3a06.pdf

Almeida, A. S. A. de (2009). Bullies, vitimas, bullies-vitimas e bystanders: A empatia e a regulação emocional da autoeficácia (Dissertação de mestrado). Departamento de Psicologia, Instituto Universitário de Lisboa. Portugal.

Analitis, F., Velderman, M. K., Ravens-Sieberer, U., Detmar, S., Erhart, M., Herdman, M., Berra, S., Alonso, J., Rajmil, L., \& the European Kidscreen Group. (2009). Being bullied: Associated factors in children and adolescents 8 to 18 years old in 11 european countries. Pediatrics, 123, 569-577. doi: 10.1542/peds.2008-0323.

Arseneault, L., Walsh, E., Trzesniewski, K., Newcombe, R., Caspi, A., \& Moffitt, T. E. (2006). Bullying victimization uniquely contributes to adjustment problems in young children: A nationally representative cohort study. Pediatrics, 118, 130-138. doi: 10.1542/peds.2005-2388

Baldry, A. C., \& Farrington, D. P. (2000). Bullies and delinquents: Personal characteristics and parental 
styles. Journal of Community \& Applied Social Psychology, 10, 17-31. doi: 10.1002/(SICI)1099-1298

Bandeira, C. M. (2009). Bullying: Autoestima e diferenças de gênero (Dissertação de mestrado). Departamento de Psicologia, Universidade Federal do Rio Grande do Sul, Porto Alegre, RS.

Bender, D., \& Lösel, F. (2011). Bullying at school as a predictor of delinquency, violence and other anti-social behaviour in adulthood. Criminal Behaviour and Mental Health, 21, 99-106. doi: 10.1002/ cbm.799

Carbone-Lopez, K., Esbense, F., \& Brick, B. (2010). The correlates and consequences of peer victimization: Gender differences in direct and indirect form of bullying. Youth Violence and Juvenile Justice, 8 , 332-350. doi:10.1177/1541204010362954

Cozby, P. C. (2003). Métodos de pesquisa em ciências do comportamento. São Paulo: Atlas.

Clark, L. A., \& Watson, D. (1995). Constructing validity: Basic issues in objective scale development. Psychological Assessment, 7, 309-319. Recuperado de http://www.personal.kent.edu/ dfresco/CRM_ Readings/Clark_and_Watson_1995.pdf

Damásio, B. F. (2012). Uso da análise fatorial exploratória em psicologia. Avaliação Psicológica, 11, 213-228. Recuperado de http://pepsic. bvsalud.org/scielo.php?script=sci_arttext\&pi$\mathrm{d}=$ S1677-04712012000200007\&lng $=$ pt\&nrm $=$ iso

Fante, C. A. Z. (2005). Fenômeno bullying: Como prevenir a violência nas escolas e educar para a paz: Campinas, SP: Verus.

Farrington, D. P. (1993). Understanding and preventing bullying. In M. Tommy \& N. Morris. Crime and justice. Chicago, IL: University of Chicago Press.

Farrington, D. P. \& Ttofi, M. M. (2009). How to reduce school bullying. Victims \& Offenders, 4, 321-326. doi: 10.1080/15564880903227255

Felix, E. D., Sharkey, J. D., Green, J. G., Furlong, M. J., \& Tanigawa, D. (2011). Getting precise and pragmatic about the assessment of bullying: The development of the California bullying victimization scale. Aggressive Behavior, 37, 234-247. doi: 10.1002/ab.20389.

Ferraz, S. F. da S. (2008). Comportamentos de bullying: Estudo numa escola técnico-profissional (Dissertação de mestrado). Departamento de Medicina, Universidade do Porto, Portugal.

Fitzpatrick, S., \& Bussey, K. (2011). The development of the social bullying involvement scales. Aggressive Behavior, 37, 177-192. doi: 10.1002/ab.20379.

Fleming, L. C., \& Jacobsen, K. H. (2009). Bullying and symptoms of depression in chilean middle school students. Journal of School Health, 79, 130-137. doi: 10.1111/j.1746-1561.2008.0397.x

Fonseca, I., \& Veiga, F. H (2007). Violência escolar e bullying em paises europeus. Em A. Barca, M. Peralbo, A. Porto, B. Duarte da Silva \& L. Almeida (Ed.), Livro de Actas do IX Congresso Internacional Galego-Português de Psicopedagogia (pp. 107-118).

Fosse, G. K. (2006). Mental health of psychiatric outpatients bullied in childhood. Norwegian University of Science and Technology. Oslo.

Hair, J. F., Jr., Black, W. C., Babin, B. J., Anderson, R. E., \& Tatham, R. L. (2009). Análise multivariada de dados ( $6^{a}$ ed.) Porto Alegre, RS: Bookman.

Hayton, J. C., Allen, D. G., \& Scarpello, V. (2004). Factor retention decisions in exploratory factor analysis: A tutorial on parallel analysis. Organizational Research Methods, 7, 191-205. doi 10.1177/1094428104263675

Holmberg, K., \& Hjern, A. (2008). Bullyng and attention-deficit-hyperactivity disorder in 10-yerolds in a Swedish community. Developmental Medicine \& Child Neurology, 50, 134-138. doi: 10.1111/j.1469-8749.2007.02019.x

Ireland, J. L., \& Archer, J. (2004). Association between measures of aggression and bullying among juvenile and young offenders. Aggressive Behavior, 30, 29-42. doi: 10.1002/ab.20007

Jimerson, S. R., Swearer, S. M., \& Espelage, D. L. (2010). Handbook of bullying in schools: A international perspective. New York, US: Routledge.

Juvonen, J., \& Gross, E. F. (2008). Extending the school grounds? Bullying experiences in cyberspace. Journal of School Health, 78, 496-505. doi: 10.1111/j.1746-1561.2008.00335.x.

Kokkinos, C. M., \& Panayiotou, G. (2004). Predicting bullying and victimization amongearly adolescents: Associations with disruptive behavior disorders. Agressive Behavior, 30, 520-533. doi: 10.1002/ab.20055

Psico-USF, Bragança Paulista, v. 20, n. 3, p. 385-397, set./ dez. 2015 
Kwan, G. C. E., \& Skoric, M. M. (2013). Facebook bullying: An extension of battles in school. Computers in Human Behaviors, 29, 16-25. doi:10.1016/j. chb.2012.07.014

Kim, Y. S., Koh, Y. J., \& Leventhal, B. (2005). School bullying and suicidal risk in korean middle school students. Pediatrics, 115, 357-363. doi: 10.1542/ peds.2004-0902

Klomek, A. B., Sourander, A., \& Gould, M. (2010). The association of suicide and bullying in childhood to young adulthood: A review of cross-sectional and longitudinal research findings. The Canadian Journal of Psychiatry, 55, 282-288. Recuperado de http:// www.ncbi.nlm.nih.gov/pubmed/20482954

Kumpulainen, K., Räsänen, E., \& Puura, K. (2001). Psychiatric disorders and the use of mental health services among children involved in bullying. $A g$ gressive Behavior, 27, 102-110. doi: 10.1002/ab.3

Lam, L. T., \& Li, Y. (2013). The validation of the evictimization scale (E-VS) and the e-bullying scale (E-BS) for adolescents. Computers in Human Behavior, 29, 3-7. doi:10.1016/j.chb.2012.06.021

Lemos, A. C. M. (2007). Uma visão psicopedagógica do bullying escolar. Revista Psicopedagogia, 24, 68-75. Recuperado de http://pepsic.bvsalud.org/pdf/ psicoped/v24n73/v24n73a09.pdf

Lopes Neto, A. A. (2005). Bullying: Comportamento agressivo entre estudantes. Jornal de Pediatria, 81, 164-172. Recuperado de http://www.uff.br/saudecultura/encontros/Bullyng.pdf

Maidel, S. (2009). Cyberbullying: Um novo risco advindo das tecnologias digitais. Revista electrónica de investigación y docência, 2,113-119. Recuperado de http://www.ujaen.es/revista/reid/revista/n2/ REID2art7.pdf

Méndez, I., \& Cerezo, F. (2010). Bullying y factores de riesgo para la salud en estudiantes de secundaria. European Journal of Education and Psychology, 3, 209-218. Recuperado de http://www.redalyc.org/ articulo.oa?id $=129315468005$

Monks, C. P., \& Coyne, I. (2011). Bullying in Different Contexts. Cambridge: Cambridge University Press.

Moura, D. R., Cruz, A. N. C., \& Quevedo, L. A. (2011). Prevalence and characteristics of school age bullying victims. Jornal de Pediatria, 87, 19-23. doi: 10.1590/S0021-75572011000100004
Nunnally, J. C. (1991). Teoría psicométrica. México, DF: Trillas.

Olweus, D. (1993). Bullyng at school: What we know and what we can do. Cambridge, MA: Blackwell.

Olweus, D. (2003). A profile of bullying at school. Educational Leadership, 60, 12-17. Recuperado de http://www.ascd.org/publications/ educational-leadership/mar03/vol60/num06/AProfile-of-Bullying-at-School.aspx

Olweus, D. (2011). Bullying at school and later criminality: Findings from three Swedish community samples of males. Criminal Behaviour and Mental Health, 21, 151-156. doi: 10.1002/cbm.806.

Owusu, A., Hart, P., Oliver, B., \& Kang, M (2011). The association between bullying and psychological health among senior high school students in Ghana, West Africa. Journal of School Health, 81, 231-238. doi: 10.1111/j.1746-1561.2011.00590.x

Pasquali, L. (2003). Psicometria: Teoria dos testes na psicologia e na educaşão. Petrópolis, RJ: Vozes.

Pasquali, L. (2010). Instrumentação psicológica: Fundamentos epráticas. Porto Alegre, RS: Artmed.

Pasquali, L. (2012). Análise fatorial para pesquisadores. Brasilia, DF: LabPam.

Pinheiro, F. M. F. (2006). Violência intrafamiliar e envolvimento em "bullying" no ensino fundamental. Dissertação de mestrado não publicada. Universidade Federal de São Carlos, São Carlos, SP.

Ribeiro, A. T. M. (2007). O bullying em contexto escolar estudo de caso. Dissertação de mestrado não publicada. Universidade Portucalense, Portugal.

Rivers, I., \& Smith, P. K. (1994). Types of bullying behaviour and their correlates. Aggressive Behavior, 20, 359-368. doi: 10.1002/1098-2337

Rolim, M. (2008). Bullying: O pesadelo da escola. Um estudo de caso e notas sobre o que fazerer (Dissertação de mestrado). Departamento de Sociologia, Universidade Federal do Rio Grande do Sul, Porto Alegre, RS.

Salmivalli, C. (2010). Bullying and the peer group: A review. Agression and Violent Behavior, 15, 112-120. doi:10.1016/j.avb.2009.08.007

Sanders, C. E. (2004). What is Bullying? In C. E. Sanders \& G. D. Phye. Bullying: Implications for the classroom (pp. 2-18). San Diego, CA: Elsevier Academic Press. 
Santos, L. C. O., Gouveia, R. S. V., Soares, A. K. S., Cavalcanti, T. M., \& Gouveia, V. V. (no prelo). Forms of bullying scale (FBS): Evidências de validade de construto da versão brasileira. Avaliação Psicológica.

Scheithauer, H., Hayer, T., Petermann, F., \& Jugert, G. (2006). Physical, verbal, and relational forms of bullying among german students: Age trends, gender differences, and correlates. Aggressive Behavior, 32, 361-375. doi: 10.1002/ab.20128

Schoffstall, C. L., \& Cohen, R. (2011). Cyber aggression: The relation between online offenders and offline social competence. Social Development, 20, 587-604. doi: 10.1111/j.1467-9507.2011.00609.x

Shaw, T., Dooley, J. J., Cross, D., Zubrick, S. R., \& Waters, S. (2013). The forms of bullying scale (FBS): Validity and reliability estimates for a measure of bullying victimization and perpetration in adolescence. Psychological Assessment, 25, 1045-1057. doi: $10.1037 / \mathrm{a} 0032955$

Skapinakis, P., Bellos S., Gkatsa, T., Magklara, K., Lewis, G., Araya, R., Mavreas, V., \& Stylianidis, S. (2011).The association between bullying and early stages of suicidal ideation in late adolescents in Greece. BMC Psychiatry, 11, 1-9. doi:10.1186/ 1471-244X-11-22

Slonje, R., \& Smith, P. K. (2008). Cyberbullying: Another main type of bullying? Scandinavian Journal of Psychology, 49, 147-154. doi: 10.1111/j.1467-9450.2007.00611.x

Smith, P. K., Mahdavi, J., Carvalho, M., Fischer, S., Russell S., \& Tippett, N. (2008). Cyberbullying: Its nature and impact in secondary school pupils. Journal of Child Psychology and Psychiatry, 49, 376-385. doi: 10.1111/j.1469-7610.2007.01846.x

Soares, A. K. S. (2013). Valores bumanos e bullying: Um estudo pautado na congruência entre pais e filhos. Dissertação de Mestrado. Departamento de Psicologia, Universidade Federal da Paraíba, João Pessoa, PB.

Solberg, M. E., Olweus, D., \& Endresen, I. M. (2007). Bullies and victims at school: Are they the same pupils? British Journal of Educational Psychology, 77, 441-464. Recuperado de http://www.ncbi.nlm. nih.gov/pubmed/17504556

Sourander, A. Jensen, P., Rönning, J. A., Elonheimo, H., Niemela, S. Helenius, H., Kumpulainen, K., Piha, J.
Tamminen, T., Moilanen, I., \& Almqvist, F. (2007). Childhood bullies and victims and their risk of criminality in late adolescence. Archives Pediatricsand Adolescent, 161, 546-552. Recuperado de http:// www.ncbi.nlm.nih.gov/pubmed/17548758

Thomas, H. J., Connor, J. P., \& Scott, J. G. (2015). Integrating traditional bullying and cyberbullying: Challenges of definition and measurement in adolescents - a review. Educational Psychology Review, 27, 135-152. doi: 10.1007/s10648-014-9261-7

Urbina, S. (2007). Fundamentos da testagem psicológica. Porto Alegre: Artmed.

Vandebosch, H., \&Van Cleemput, K. (2008). Defining cyberbullying: A qualitative research into the perceptions of youngsters. Cyberpsychology Behavior, 11, 499-503. doi: 10.1089/cpb.2007.0042

Vessey, J., Strout, T. D., DiFazio, R. L., \& Walker, A. (2014). Measuring the youth bullying experience: A systematic review of the psychometric properties of available instruments. Journal of School Health, 82, 819-843. doi: 10.1111/josh.12210

Wal, M. F. Van der (2005). There is bullying and bullying. European Journal of Pediatrics, 164, 117118. Recuperado de http://link.springer.com/ article/10.1007\%2Fs00431-004-1573-z

Wal, M. F. Van der, Wit, C. A. M. de, Hirasing, R. A. (2003). Psychosocial health among young victims and offenders of direct and indirect bullying. Pediatrics, 111, 1312-1317. Recuperado de http:/ /www. ncbi.nlm.nih.gov/pubmed/12777546

Wang, W., Iannotti, R. J., \& Nansel, T. R. (2009). School bullying among US adolescents: Physical, verbal, relational and cyber. Journal Adolescent Health, 45, 368-375. doi: 10.1016/j.jadohealth.2009.03.021

Wolke, D., Woods, S., Bloomfield, L., \& Karstadt, L. (2000). The association between direct and relational bullying and behavior problems among primary school children. Journal of Child Psychology and Psychiatry, 41, 989-1002. Recuperado de http:// www.ncbi.nlm.nih.gov/pubmed/11099116

Recebido: 04/07/2014 Primeira reformulação: 10/02/2015 Segunda reformulação: 13/03/2015 Aprovado: 20/03/2015 
Nota dos autores:

Os autores aproveitam a oportunidade para agradecer a seguintes instituições: ao CNPq que financiou o projeto do primeiro autor, do qual é oriundo este estudo, por meio do Edital Universal 14/2011, proc. 479738/2011-4 e por conceder bolsa de produtividade ao segundo autor; e à CAPES, por conceder bolsa de mestrado ao terceiro autor.

Sobre os autores:

Emerson Diógenes de Medeiros é psicólogo, mestre e doutor em Psicologia Social pela UFPB, atualmente, professor adjunto do curso de graduação em Psicologia da Universidade Federal do Piauí (Campus de Parnaíba) e coordena o Laboratório de Avaliação Psicológica do Delta - LABAP. Seus interesses de pesquisa centram-se em Psicologia Social (valores humanos e atitudes), construção e adaptação de medidas psicológicas.

E-mail: emersondiogenes@gmail.com

Valdiney Veloso Gouveia é licenciado (1989) e possui formação (2005) em Psicologia pela Universidade Federal da Paraíba, especialista em Psicometria (1993), mestre em Psicologia Social e do Trabalho (1991) pela Universidade de Brasília e doutor em Psicologia Social (1998) pela Universidade Complutense de Madri. Atualmente é professor titular na Universidade Federal da Paraíba.

E-mail:vvgouveia@gmail.com

Renan Pereira Monteiro possui formação em Psicologia pela Universidade Federal do Piauí (2011), é mestre em Psicologia Social pela Universidade Federal da Paraíba (2014) e, atualmente, é doutorando em Psicologia Social pela Universidade Federal da Paraíba. Seus interesses de pesquisa centram-se na avaliação da personalidade (traços normais e aversivos), Psicologia Social (valores humanos e atitudes), construção e adaptação de medidas psicológicas.

E-mail: renanpmonteiro@gmail.com

Paulo Gregório Nascimento da Silva é graduado em Psicologia pela Universidade Federal do Piauí (2014) e integrante do Laboratório de Avaliação Psicológica do Delta (LABAP-D). Tem interesse em construção e validade de testes, escalas e outras medidas psicológicas.

E-mail: silvapgn@gmail.com

Bruna de Jesus Lopes é formada em Psicologia pela Universidade Federal do Piauí - UFPI e, atualmente, é mestranda em Psicologia Social pela Universidade Federal da Paraíba.

E-mail:bruna_lopespsi@hotmail.com

Paloma Cavalcante Bezerra de Medeiros é graduada em Psicologia nas habilitações licenciatura e formação de Psicólogo pela Universidade Federal da Paraíba (UFPB), mestre em Psicologia Social pelo programa de Pós-Graduação em Psicologia Social da UFPB, atualmente, é doutoranda nesse mesmo programa e professora assistente do Departamento de Psicologia da Universidade Federal do Piauí. Sua principal experiência é na área de Neurociência Social.

E-mail:palomacbemedeiros@gmail.com

Élido Santiago da Silva é mestre em Administração pelo PPGA/UFRN (2010) e graduado em Pedagogia pela Universidade do Estado do Pará (2003). Tem experiência nas áreas de Educação, com ênfase em gestão educacional, e de Administração, com ênfase em Gestão de Recursos Humanos. Atualmente é professor assistente I da Universidade Federal do Piauí.

E-mail: elidosantiago@gmail.com

Contato com os autores:

Prof. Emerson Diógenes de Medeiros

E-mail: emersondiogenes@gmail.com

Universidade Federal do Piauí

Departamento de Psicologia

Laboratório de Avaliação Psicológica do Delta - LABAP

Av. São Sebastião, 2819 - Parnaíba/PI.

CEP: 64202-020

Psico-USF, Bragança Paulista, v. 20, n. 3, p. 385-397, set./ de₹. 2015 
